\title{
Observation of resonance condensation of fermionic atom pairs
}

\author{
C. A. Regal, M. Greiner, and D. S. Jin $*$ \\ JILA, National Institute of Standards and Technology and University of Colorado, \\ and Department of Physics, University of Colorado, Boulder, CO 80309-0440
}

(Dated: January 13, 2004)

\begin{abstract}
We have observed condensation of fermionic atom pairs in the BCS-BEC crossover regime. A trapped gas of fermionic ${ }^{40} \mathrm{~K}$ atoms is evaporatively cooled to quantum degeneracy and then a magnetic-field Feshbach resonance is used to control the atom-atom interactions. The location of this resonance is precisely determined from low-density measurements of molecule dissociation. In order to search for condensation on either side of the resonance we introduce a technique that pairwise projects fermionic atoms onto molecules; this enables us to measure the momentum distribution of fermionic atom pairs. The transition to condensation of fermionic atom pairs is mapped out as a function of the initial atom gas temperature $T$ compared to the Fermi temperature $T_{F}$ for magnetic-field detunings on both the BCS and BEC sides of the resonance.
\end{abstract}

Ultracold quantum gases of fermionic atoms with tunable interactions offer the unique possibility to experimentally access the predicted crossover between BCStype superfluidity of momentum pairs and Bose-Einstein condensation (BEC) of molecules [1, 2, 3, 4, 5, 6, 7]. Magnetic-field Feshbach resonances provide the means for controlling both the magnitude of cold atom interactions, characterized by the s-wave scattering length $a$, as well as whether they are, in the mean-field approximation, effectively repulsive $(a>0)$ or attractive $(a<0)$ [8, 9]. For magnetic-field detunings on the $a>0$, or BEC, side of the resonance there exists an extremely weakly bound molecular state whose binding energy depends strongly on the detuning from the Feshbach resonance 10, 11. In Fermi gases this state can be long lived [12, 13, 14, 15]. BEC of these molecules represents one extreme of the predicted BCS-BEC crossover and recently has been observed for both ${ }^{40} \mathrm{~K}_{2}$ and ${ }^{6} \mathrm{Li}_{2}$ molecules [16, 17, 18].

In atomic Fermi gas systems condensates have not previously been observed beyond this molecular BEC extreme [19]. In discussing condensation of a Fermi gas throughout the BCS-BEC crossover, terms such as Cooper pairs, molecules, BEC, and fermionic condensates often have ambiguous meanings. In this paper we define "condensation of fermionic atom pairs", or equivalently fermionic condensates, as a condensation (i.e. the macroscopic occupation of a single quantum state) in which the underlying Fermi statistics of the paired particles plays an essential role 20. In the BCS extreme this is more commonly termed condensation of Cooper pairs. Fermionic condensates are distinct from the BEC extreme where there remains no fermionic degree of freedom because all fermions are bound into bosonic molecules 21]. In this Letter we report the observation of condensation of fermionic atom pairs near and on both sides of the Feshbach resonance, which corresponds to the BCS-BEC crossover regime. We observe condensation on the $a<0$, or BCS, side of the Feshbach resonance. Here the two-body physics of the resonance no longer supports the weakly bound molecular state; hence, only cooperative many-body effects can give rise to this condensation of fermion pairs [4, 5, 6, 7, 22] .

Demonstrating condensation of fermionic atom pairs on the BCS side of the resonance presents significant challenges. Observation of pairing of fermions 23] is insufficient to demonstrate condensation, and rather a probe of the momentum distribution is required [7]. For example, the standard technique developed for observing BEC relies on time-of-flight expansion images [24, 25]. However, this method is problematic on the BCS side of the resonance because the pairs depend on many-body effects and are not bound throughout expansion of the gas. In this work we introduce a technique that takes advantage of the Feshbach resonance to pairwise project the fermionic atoms onto molecules. We probe the system by rapidly

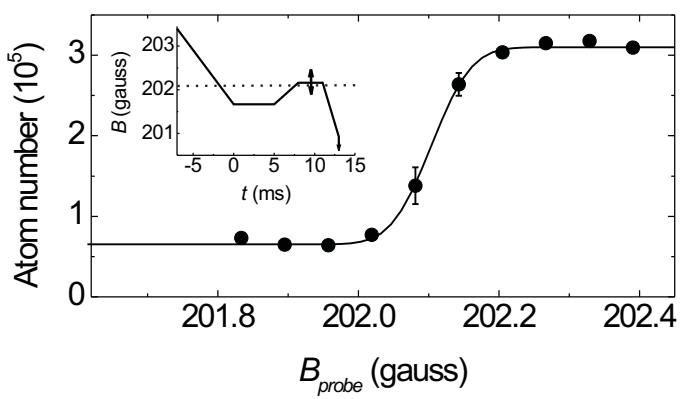

FIG. 1: Measurement of the Feshbach resonance position $B_{0}$. Shown in the inset is a schematic of the magnetic field as a function of time $t$ measured with respect to the optical trap turn off at $t=0$. Molecules are first created by a slow magnetic-field sweep across the resonance (dotted line) and then dissociated if $B_{\text {probe }}$ (indicated by the arrow in the inset) is beyond the magnetic field where the two-body physics supports a new bound state. The number of atoms, measured at $t=17 \mathrm{~ms}$, is shown as a function of $B_{\text {probe. The }}$ two error bars indicate the spread in repeated points at these values of $B$. A fit of the data to an error function reveals $B_{0}=202.10 \pm 0.07 \mathrm{G}$, where the uncertainty is given conservatively by the $10 \%-90 \%$ width. 


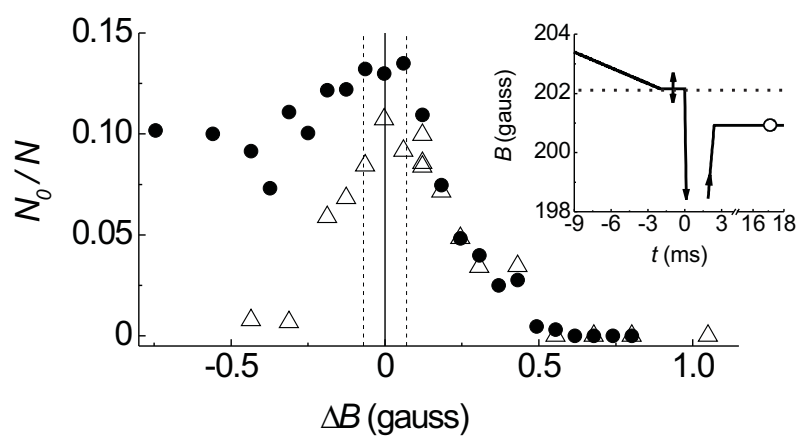

FIG. 2: Measured condensate fraction as a function of detuning from the Feshbach resonance $\Delta B=B_{\text {hold }}-B_{0}$. Data here were taken for $t_{\text {hold }}=2 \mathrm{~ms}(\bullet)$ and $t_{\text {hold }}=30 \mathrm{~ms}(\triangle)$ with an initial cloud at $T / T_{F}=0.08$ and $T_{F}=0.35 \mu \mathrm{K}$. The area between the dashed lines around $\Delta B=0$ reflects the uncertainty in the Feshbach resonance position based on the $10 \%-90 \%$ width of the feature in Fig. 1] Condensation of fermionic atom pairs is seen near and on either side of the Feshbach resonance. Comparison of the data taken with the different hold times indicates that the pair condensed state has a significantly longer lifetime near the Feshbach resonance and on the BCS $(\Delta B>0)$ side. The inset shows a schematic of a typical magnetic-field sweep used to measure the fermionic condensate fraction. The system is first prepared by a slow magnetic-field sweep towards the resonance (dotted line) to a variable position $B_{\text {hold }}$, indicated by the two-sided arrow. After a time $t_{\text {hold }}$ the optical trap is turned off and the magnetic field is quickly lowered by $\sim 10 \mathrm{G}$ to project the atom gas onto a molecular gas. After free expansion, the molecules are imaged on the BEC side of the resonance (o).

sweeping the magnetic field to the $a>0$, or BEC, side of the resonance, where time-of-flight imaging can be used to measure the momentum distribution of the weakly bound molecules. The projecting magnetic-field sweep is completed on a timescale that allows molecule formation but is still too brief for particles to collide or move significantly in the trap. This projection always results in $60 \%$ to $80 \%$ of the atom sample appearing as molecules. However, we find that there is a threshold curve of $T / T_{F}$ versus detuning from the Feshbach resonance below which we observe a fraction of the molecules to have near zero momentum. We interpret this as reflecting a pre-existing condensation of fermionic atom pairs.

Our basic experimental procedures have been discussed in prior work 26, 27]. We trap and cool a dilute gas of the fermionic isotope ${ }^{40} \mathrm{~K}$, which has a total atomic spin $f=9 / 2$ in its lowest hyperfine ground state and thus ten available Zeeman spin-states $\left|f, m_{f}\right\rangle$ 26, 28]. We use a far-off resonance optical dipole trap that can confine atoms in any spin state as well as the molecules we create from these atoms. The optical trap is characterized by radial frequencies ranging between $\nu_{r}=320$ and $440 \mathrm{~Hz}$, with the trap aspect ratio, $\nu_{r} / \nu_{z}$, fixed at $79 \pm 15$.

Experiments are initiated by preparing atoms in a

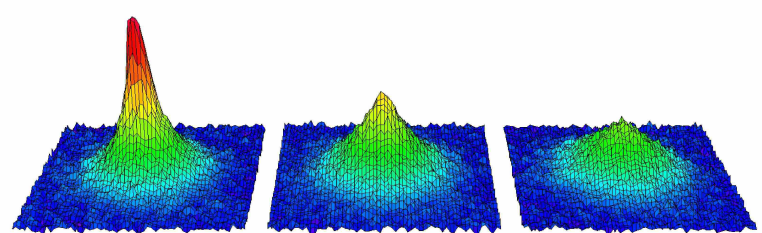

FIG. 3: Time of flight images showing the fermionic condensate. The images, taken after the projection of the fermionic system onto a molecular gas, are shown for $\Delta B=0.12,0.25$, and $0.55 \mathrm{G}$ (left to right) on the BCS side of the resonance. The original atom cloud starts at $T / T_{F}=0.07$, and the resulting fitted condensate fractions are $N_{0} / N=0.10,0.05$, and 0.01 (left to right). Each image corresponds to $N=100,000$ particles and is an average over 10 cycles of the experiment.

nearly equal, incoherent mixture of the $|9 / 2,-7 / 2\rangle$ and $|9 / 2,-9 / 2\rangle$ spin states at a low $T / T_{F}$. We access an swave Feshbach resonance between these states located at a magnetic field near $200 \mathrm{G}$ [29]. A precise determination of the magnetic-field location of the two-body resonance is an essential ingredient for exploring the BCSBEC crossover regime. In our previous work the location of the resonance was determined from the peak in the resonantly enhanced elastic collision rate $15,30,31$. In the work reported here we have more precisely determined the location of the resonance by measuring the magnetic field, $B_{0}$, above which the two-body physics no longer supports the shallow bound state.

Figure 1] shows the result of such a measurement. Molecules created by a slow magnetic-field sweep across the resonance are dissociated by raising the magnetic field to a value $B_{\text {probe }}$ near the resonance (inset to Fig. 1). To avoid many-body effects, this dissociation occurs after allowing the gas to expand from the trap to much lower density. After a total expansion time of $17 \mathrm{~ms}$ atoms not bound in molecules are selectively detected at near zero field [11]. The measured number of atoms increases sharply at $B_{0}=202.10 \pm 0.07 \mathrm{G}$. This more precise measurement of the resonance position agrees well with previous results [15, 30, 31]. As an additional check, we have located the resonance by creating, rather than dissociating, molecules. The measured number of molecules decreases sharply at $B=202.14 \pm 0.11 \mathrm{G}$ in good agreement with the molecule dissociation result [32].

In order to investigate the BCS-BEC crossover regime we initially prepare the ultracold two-component atom gas at a magnetic field of $235.6 \mathrm{G}$, far above the Feshbach resonance. Here the gas is not strongly interacting, and we measure $T / T_{F}$ through surface fits to time-of-flight images of the Fermi gas 26, 27]. The field is then slowly lowered at typically $10 \mathrm{~ms} / \mathrm{G}$ to a value $B_{\text {hold }}$ near the resonance. This sweep is slow enough to allow the atoms and molecules sufficient time to move and collide in the trap. This was shown previously in Ref. [16] where, for a 
Fermi gas initially below $T / T_{F}=0.17$, a magnetic-field sweep at $10 \mathrm{~ms} / \mathrm{G}$ to a final $B 0.56 \mathrm{G}$ below the resonance produced a molecular condensate. In this Letter, we now explore the behavior of the sample when sweeping slowly to values of $B_{\text {hold }}$ on either side of the Feshbach resonance.

To probe the system we pairwise project the fermionic atoms onto molecules and measure the momentum distribution of the resulting molecular gas. This projection is accomplished by rapidly lowering the magnetic field by $\sim 10 \mathrm{G}$ at a rate of typically $(50 \mu \mathrm{s} / \mathrm{G})^{-1}$ while simultaneously releasing the gas from the trap. This puts the gas far on the BEC side of the resonance, where it is weakly interacting. The total number of molecules after the projection, $N$, corresponds to $60 \%$ to $80 \%$ of the original atom number in each spin state. After a total of typically $17 \mathrm{~ms}$ of expansion the molecules are selectively detected using rf photodissociation immediately followed by spin-selective absorption imaging [16]. To look for condensation, these absorption images are surface fit to a two-component function that is the sum of a ThomasFermi profile for a condensate and a gaussian function for non-condensed molecules [16].

Figures 2 through 4 present the main result of this paper. In Fig. 2 we plot the measured condensate fraction $N_{0} / N$ as a function of the magnetic-field detuning from the resonance, $\Delta B=B_{\text {hold }}-B_{0}$ 33]. The data in Fig. 2 was taken for a Fermi gas initially at $T / T_{F}=0.08$ and for two different wait times at $B_{\text {hold }}$. Condensation is observed on both the BCS $(\Delta B>0)$ and $\operatorname{BEC}(\Delta B<0)$ sides of the resonance. We further find that the condensate on the BCS side of the Feshbach resonance has a relatively long lifetime. The lifetime was probed by increasing $t_{\text {hold }}$ to $30 \mathrm{~ms}$ (triangles in Fig. 2). We find that for the BEC side of the resonance no condensate is observed for $t_{\text {hold }}=30 \mathrm{~ms}$ except very near the resonance. However, for all data on the BCS side of the resonance the observed condensate fraction is still $>70 \%$ of that measured for $t_{\text {hold }}=2 \mathrm{~ms}$. Finally, we note that the appearance of the condensate is accompanied by a significant (as large as 20\% at the resonance) decrease in the measured width of the non-condensed fraction. This effect will be a subject of future investigations.

Figure 3 displays sample time-of-flight absorption images for the fermionic condensate. Figure 4 is a phase diagram created from our data; here we plot the measured condensate fraction as function of $\Delta B$ and of the initial Fermi gas degeneracy $T / T_{F}$. The condensate forms at lower initial $T / T_{F}$ with increasing $\Delta B$, an effect predicted in $4,5,6,7$.

An essential aspect of these measurements is the fast magnetic-field sweep that pairwise projects the fermionic atoms onto molecules. It is a potential concern that the condensation might occur during this sweep rather than at $B_{\text {hold }}$. However, in our previous work it was shown that a magnetic-field sweep with an inverse speed

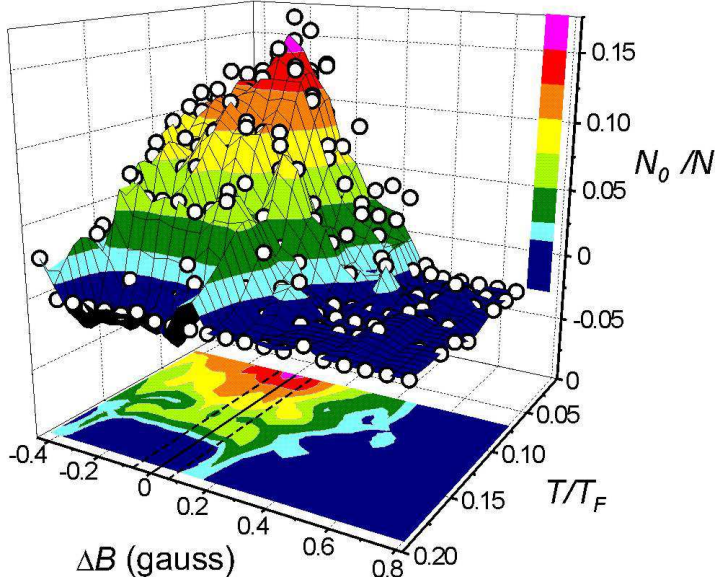

FIG. 4: Transition to condensation as a function of both $\Delta B$ and $T / T_{F}$. The data for this phase diagram were collected with the same procedure as shown in the inset to Fig. 2 with $t_{\text {hold }} \sim 2 \mathrm{~ms}$. The area between the dashed lines around $\Delta B=0$ reflects the uncertainty in the Feshbach resonance location from the width of the feature in Fig. 1. The false color surface and contour plot are obtained using a Renka-Cline interpolation of approximately 200 distinct data points (o) [34]. One measure of when the gas becomes strongly interacting is the criterion $\left|k_{F} a\right|>1$, where $\hbar k_{F}$ is the Fermi momentum 27, 35, 36, 37]. For these data, $|\Delta B|<0.6$ corresponds to $\left|k_{F} a\right|>1$.

less than $800 \mu \mathrm{s} / \mathrm{G}$ was too fast to produce a molecular condensate when starting with a Fermi gas $0.68 \mathrm{G}$ on the BCS side of the resonance 16]. Thus, the inverse sweep speed we use in this Letter of typically $50 \mu \mathrm{s} / \mathrm{G}$, while sufficiently slow to convert $60 \%$ of the sample to weakly bound molecules [1]], is much too fast to produce a molecular condensate.

In addition, we have checked that the observation of a condensate on the BCS side of the resonance does not depend on this sweep speed. As seen in Fig. 5(a), much faster sweeps result in fewer molecules. This is consistent with our previous study of the molecule creation process [1]. However, we find that the measured condensate fraction is independent of the sweep rate (Fig. 5(b)). Even with the lower number of molecules, and therefore a lower phase space density of the molecular gas, we observe an essentially unchanged condensate fraction.

Finally we note that, as in our previous measurements performed in the BEC limit, the measured condensate fraction always remains well below one [16]. As part of our probing procedure the magnetic field is dwells at $\Delta B \sim 10 \mathrm{G}$ where the molecule lifetime is only on the order of milliseconds 15, 38. This results in a measured loss of $50 \%$ of the molecules and may also reduce the measured condensate fraction. 


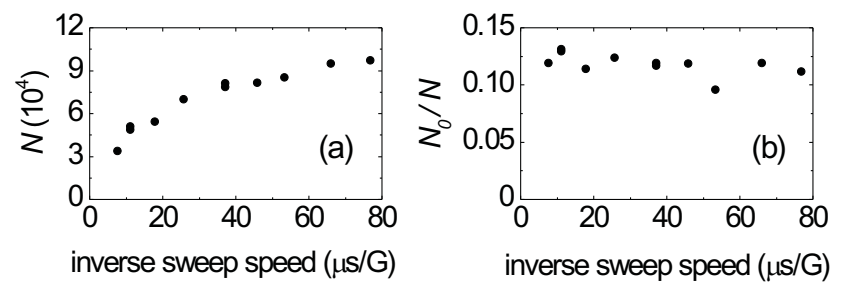

FIG. 5: Dependence of molecule number and condensate fraction on the speed of the fast magnetic-field sweep from the atomic gas onto the molecular gas. Here $\Delta B=0.12$ and the initial $T / T_{F}$ is 0.08. (a) Total number of molecules as a function of inverse sweep speed. For the fastest sweep speeds fewer molecules are created, consistent with studies in Ref. 11]. (b) Condensate fraction as a function of the inverse sweep speed. Even for the fastest sweeps and lowest molecule number, we observe an unchanged condensate fraction.

In conclusion, we have introduced a method for probing the momentum distribution of fermionic atom pairs and employed this technique to observe fermionic condensates near a Feshbach resonance. By projecting the system onto a molecular gas, we map out condensation of fermionic pairs as a function of both the magnetic-field detuning from the resonance and the initial Fermi degeneracy $T / T_{F}$. The fermionic condensates seen in this work occur in the BCS-BEC crossover regime, far from the perturbative BCS limit. As predicted, the system is observed to vary smoothly in the BCS-BEC crossover regime. Further, the lifetime of the condensed state is found to be significantly longer in the crossover regime than it is in the BEC limit. As in the case of BEC, one expects the resonance fermionic condensation observed here to correspond to superfluidity. The experimental realization of condensation in the BCS-BEC crossover regime demonstrated in this Letter follows more than two decades of theoretical investigation and initiates experimental study of this physics.

We thank E. A. Cornell, C. E. Wieman, M. Holland, K. Levin, E. Altman, and L. Radzihovsky for stimulating discussion and J. T. Smith for experimental assistance. This work was supported by NSF and NIST; C. A. R. acknowledges support from the Hertz Foundation.

* Quantum Physics Division, National Institute of Standards and Technology.

[1] A. J. Leggett, J. Phys. C. (Paris) 41, 7 (1980).

[2] P. Nozieres and S. Schmitt-Rink, J. Low-Temp. Phys. 59, 195 (1985).

[3] M. Randeria, in Bose-Einstein Condensation, edited by A. Griffin, D. W. Snoke, and S. Stringari (Cambridge University, Cambridge, 1995), pp. 355-392.

[4] M. Holland, S. J. J. M. F. Kokkelmans, M. L. Chiofalo, and R. Walser, Phys. Rev. Lett. 87, 120406 (2001).
[5] E. Timmermans, K. Furuya, P. W. Milonni, and A. K. Kerman, Phys. Lett. A 285, 228 (2001).

[6] Y. Ohashi and A. Griffin, Phys. Rev. Lett. 89, 130402 (2002).

[7] J. Stajic et al., cond-mat/0309329 (2003).

[8] W. C. Stwalley, Phys. Rev. Lett. 37, 1628 (1976).

[9] E. Tiesinga, B. J. Verhaar, and H. T. C. Stoof, Phys. Rev. A 47, 4114 (1993).

[10] E. A. Donley, N. R. Claussen, S. T. Thompson, and C. E. Wieman, Nature 417, 529 (2002).

[11] C. A. Regal, C. Ticknor, J. L. Bohn, and D. S. Jin, Nature 424, 47 (2003).

[12] K. E. Strecker, G. B. Partridge, and R. G. Hulet, Phys. Rev. Lett. 91, 080406 (2003).

[13] J. Cubizolles et al., Phys. Rev. Lett. 91, 240401 (2003).

[14] S. Jochim et al., Phys. Rev. Lett 91, 240402 (2003).

[15] C. A. Regal, M. Greiner, and D. S. Jin, Phys. Rev. Lett. in press (2004).

[16] M. Greiner, C. A. Regal, and D. S. Jin, Nature 426, 537 (2003).

[17] S. Jochim et al., Science 302, 2101 (2003).

[18] M. W. Zwierlein et al., Phys. Rev. Lett. 91, 250401 (2003).

[19] Several groups have reported attaining Fermi gases on the BCS side of a Feshbach resonance at $T / T_{F}$ below theoretical predictions for the critical condensation temperature; so far there has been no experimental evidence for condensate formation [12, 13, 27, 35, 39].

[20] H. T. C. Stoof, M. Houbiers, C. A. Sackett, and R. G. Hulet, Phys. Rev. Lett. 76, 10 (1996).

[21] We reserve the term molecule for a two-body bound state.

[22] See also: R. A. Duine and H. T. C. Stoof, J. Opt. B 5, S212 (2003).

[23] M. Greiner et al., cond-mat/0308519 (2003).

[24] M. H. Anderson et al., Science 269, 198 (1995).

[25] K. B. Davis et al., Phys. Rev. Lett. 75, 3969 (1995).

[26] B. DeMarco and D. S. Jin, Science 285, 1703 (1999).

[27] C. A. Regal and D. S. Jin, Phys. Rev. Lett. 90, 230404 (2003).

[28] G. Roati, F. Riboli, G. Modugno, and M. Inguscio, Phys. Rev. Lett. 89, 150403 (2002).

[29] J. L. Bohn, Phys. Rev. A 61, 053409 (2000).

[30] T. Loftus et al., Phys. Rev. Lett. 88, 173201 (2002).

[31] C. A. Regal, C. Ticknor, J. L. Bohn, and D. S. Jin, Phys. Rev. Lett. 90, 053201 (2003).

[32] We calibrate the magnetic field for gases in the trap and during expansion using rf transitions between Zeeman levels. The magnetic field is reproducible to $<15 \mathrm{mG}$. Mean-field shifts near the resonance are avoided through transfer between the resonant states [40].

[33] The scattering length corresponding to $\Delta B$ can be calculated from $a=a_{b g}\left(1-\frac{w}{\Delta B}\right)$, where $a_{b g}=174 a_{0}$ and $w=7.8 \pm 0.6 \mathrm{G}[31]$.

[34] R. L. Renka and A. K. Cline, Rocky Mountain J. Math 14, 223 (1984).

[35] K. M. O'Hara et al., Science 298, 2179 (2002).

[36] T. Bourdel et al., Phys. Rev. Lett. 91, 020402 (2003).

[37] S. Gupta et al., Science 300, 1723 (2003).

[38] D. S. Petrov, C. Salomon, and G. V. Shlyapnikov, cond-mat/0309010 (2003).

[39] M. Bartenstein et al., cond-mat/0401109 (2004).

[40] M. W. Zwierlein, Z. Hadzibabic, S. Gupta, and W. Ketterle, Phys. Rev. Lett. 91, 250404 (2003). 